BARI-TH 398/00

DFF-365/10/00

UGVA-DPT-2000/09-1089

\title{
Dispersion Laws for In-medium Fermions and Gluons in the CFL Phase of QCD
}

\author{
R. Casalbuoni ${ }^{a, b}$, R. Gatto ${ }^{c}$ and G. Nardullid,e \\ ${ }^{a}$ Dipartimento di Fisica, Università di Firenze, I-50125 Firenze, Italia \\ ${ }^{b}$ I.N.F.N., Sezione di Firenze, I-50125 Firenze, Italia \\ ${ }^{c}$ Départ. de Physique Théorique, Université de Genève, CH-1211 Genève 4, \\ Suisse \\ ${ }^{d}$ Dipartimento di Fisica, Università di Bari, I-70124 Bari, Italia \\ ${ }^{e}$ I.N.F.N., Sezione di Bari, I-70124 Bari, Italia
}

\begin{abstract}
We evaluate several quantities appearing in the effective lagrangian for the color-flavor locked phase of high density QCD using a formalism which exploits the approximate decoupling of fermions with energy negative with respect to the Fermi energy. The effective theory is essentially two-dimensional and exhibits a Fermi velocity superselection rule, similar to the one found in the Heavy Quark Effective Theory. Within the formalism we reproduce, using gradient expansion, the results for the effective parameters of the Nambu-Goldstone bosons. We also determine the dispersion laws for the gluons. By coupling the theory to fermions and integrating over the two-dimensional degrees of freedom we obtain the effective description of in-medium fermions.
\end{abstract}

\section{Introduction}

QCD at high density is expected to be in a chirality breaking color superconducting phase due to formation of quark Cooper pairs [1], [2]. In QCD with three massless quarks one expects simultaneous breaking of color and flavor 
simmetries into a residual diagonal color-flavor locked symmetry (CFL). The physical description and consequences have been discussed by a number of authors [3, 4, 5, 6]. A formalism has been recently proposed [7] and [8] to deal with the color superconducting phase. It takes advantage of the approximate decoupling of the states of a massless fermion of energy distant with respect to the Fermi surface. We shall first summarize the main consequences of such a description, with emphasis on its implied velocity superselection rule, similar to that familiar in the Heavy Quark Effective Theory (HQET) [9], and on its essentially two-dimensional features, due to the special relevance of momenta parallel to the Fermi velocity. We shall then calculate in this formalism the effective parameters of the goldstones, reobtaining results derived by other methods. Next we shall calculate the dispersion laws for the gluons and discuss the various masses that can be defined for the scalar, longitudinal and transverse gluons, i.e. their rest mass, the effective mass and the inverse of the penetration length. After having coupled the theory to fermions we obtain the effective description of in-medium fermions, by integrating over the two-dimensional degrees of freedom.

In section 2 we discuss the effective theory on the Fermi surface. In section 3 we write down the effective lagrangian for the fermions and the goldstones. In section 4 we perform the gradient expansion on the generating functional. We discuss the gluon polarization tensor in section 5. In section 6 we treat the effective description of in-medium fermions.

\section{Effective theory on the Fermi surface}

As discussed in the introduction, we will make use of the formalism developed in refs. [7, 8] to evaluate several quantities appearing in the effective lagrangian [4] describing the CFL phase of QCD at high density. This formulation is based on the observation that, at very high-density, the energy spectrum of a massless fermion is described by states $| \pm\rangle$ with energies

$$
E_{ \pm}=-\mu \pm|\vec{p}|
$$

where $\mu$ is the is the quark number chemical potential. For energies much lower than the Fermi energy $\mu$, only the states $|+\rangle$ close to the Fermi surface. i.e. with $|\vec{p}| \approx \mu$, can be excited. On the contrary, the states $|-\rangle$ have $E_{-} \approx-2 \mu$ and therefore they decouple leaving in the physical spectrum only 
the states $|+\rangle$ and the gluons. This can be seen more formally by writing the four-momentum of the fermion as

$$
p^{\mu}=\mu v^{\mu}+\ell^{\mu},
$$

where $v^{\mu}=\left(0, \vec{v}_{F}\right)$, with $\left|\vec{v}_{F}\right|=1$. Since the hamiltonian for a massless Dirac fermion in a chemical potential $\mu$ is

$$
H=-\mu+\vec{\alpha} \cdot \vec{p}, \quad \vec{\alpha}=\gamma_{0} \vec{\gamma},
$$

one has

$$
H=-\mu\left(1-\vec{\alpha} \cdot \vec{v}_{F}\right)+\vec{\alpha} \cdot \vec{\ell}
$$

Then, it is convenient to introduce the projection operators

$$
P_{ \pm}=\frac{1 \pm \vec{\alpha} \cdot \vec{v}_{F}}{2}
$$

such that

$$
H|+\rangle=\vec{\alpha} \cdot \vec{\ell}|+\rangle, \quad H|-\rangle=(-2 \mu+\vec{\alpha} \cdot \vec{\ell})|-\rangle .
$$

In terms of fields, the momentum decomposition in eq. (2) and the projection of the states $| \pm\rangle$ can be realized through

$$
\psi(x)=\sum_{\vec{v}_{F}} e^{-i \mu v \cdot x}\left[\psi_{+}(x)+\psi_{-}(x)\right],
$$

where $\sum_{\vec{v}_{F}}$ is an average over the Fermi velocities, and the velocity-dependent fields $\psi_{ \pm}(x)$ are given by:

$$
\psi_{ \pm}(x)=e^{i \mu v \cdot x}\left(\frac{1 \pm \vec{\alpha} \cdot \vec{v}_{F}}{2}\right) \psi(x)=\int_{|\ell|<\mu} \frac{d^{4} \ell}{(2 \pi)^{4}} e^{-i \ell \cdot x} \psi_{ \pm}(\ell) .
$$

Introducing the four-vectors

$$
V^{\mu}=\left(1, \vec{v}_{F}\right), \quad \tilde{V}^{\mu}=\left(1,-\vec{v}_{F}\right),
$$

it is easy to derive the following identities

$$
\begin{aligned}
& \bar{\psi}_{+} \gamma^{\mu} \psi_{+}=\psi_{+}^{\dagger} V^{\mu} \psi_{+} \\
& \bar{\psi}_{-} \gamma^{\mu} \psi_{-}=\psi_{-}^{\dagger} \tilde{V}^{\mu} \psi_{-} \\
& \bar{\psi}_{+} \gamma^{\mu} \psi_{-}=\bar{\psi}_{+} \gamma_{\perp}^{\mu} \psi_{-} \\
& \bar{\psi}_{-} \gamma^{\mu} \psi_{+}=\bar{\psi}_{-} \gamma_{\perp}^{\mu} \psi_{+} .
\end{aligned}
$$


where

$$
\gamma_{\perp}^{\mu}=\frac{1}{2} \gamma_{\nu}\left(2 g^{\mu \nu}-V^{\mu} \tilde{V}^{\nu}-\tilde{V}^{\mu} V^{\nu}\right) .
$$

Substituting into the Dirac part of the QCD lagrangian density one obtains

$$
\mathcal{L}=\sum_{\vec{v}_{F}}\left[\psi_{+}^{\dagger} i V \cdot D \psi_{+}+\psi_{-}^{\dagger}(2 \mu+i \tilde{V} \cdot D) \psi_{-}+\left(\bar{\psi}_{+} i \not D_{\perp} \psi_{-}+\text {h.c. }\right)\right],
$$

where $\not_{\perp}=D_{\mu} \gamma_{\perp}^{\mu}$ and $D_{\mu}$ is the usual covariant derivative. We notice that the fields appearing in (12) are evaluated at the same Fermi velocity because off-diagonal terms are cancelled by the rapid oscillations of the exponential factor in the $\mu \rightarrow \infty$ limit. This behaviour is similar to that in the Heavy Quark Effective Theory [9], and can be referred to, by analogy, as the Fermi velocity superselection rule.

At the leading order in $1 / \mu$ one has

$$
i V \cdot D \psi_{+}=0, \quad \psi_{-}=-i \frac{1}{2 \mu} \gamma_{0} D_{\perp} \psi_{+},
$$

showing the decoupling of $\psi_{-}$in the $\mu \rightarrow \infty$ limit. Therefore, in this limit, the $\psi_{-}$field plays no role, except in those loop diagrams, of the order of $\mu^{3}$ instead of $\mu^{2}$, where the $1 / \mu$ factor in (13) is eaten by the extra $\mu$ factor from the momentum integration. We shall see an example in Section 5. The equation for $\psi_{+}$shows also that only the energy and the momentum parallel to the Fermi velocity are relevant variables in the problem. We have an effective two-dimensional theory.

In conclusion at the next to leading order in $\mu$ we have

$$
\mathcal{L}=\sum_{\vec{v}_{F}}\left[\psi_{+}^{\dagger} i V \cdot D \psi_{+}-\frac{1}{2 \mu} \psi_{+}^{\dagger}\left(\not D_{\perp}\right)^{2} \psi_{+}\right] .
$$

Further terms in the $1 / \mu$ expansion can be found in [7].

The previous remarks apply to any theory describing massless fermions at high density. In the case of CFL phase of $\mathrm{QCD}$, in order to implement correctly the symmetry properties, one has to consider left- and right-handed fermions transforming respectively as the $(\mathbf{3}, \mathbf{1}, \mathbf{3})$ and $(\mathbf{1}, \mathbf{3}, \mathbf{3})$ representations of $S U(3)_{L} \otimes S U(3)_{R} \otimes S U(3)_{c}$. Therefore we will consider for each leftand right-handed field an effective two-dimensional theory as outlined above. The next step will be to couple this theory in a $S U(3)_{L} \otimes S U(3)_{R} \otimes S U(3)_{c}$ 
invariant way to Nambu-Goldstone bosons (NGB) describing the appropriate breaking

$$
S U(3)_{L} \otimes S U(3)_{R} \otimes S U(3)_{c} \rightarrow S U(3)
$$

for the CFL phase. Using a gradient expansion we will get an explicit expression for the decay coupling constant of the Nambu-Goldstone boson as well as for its velocity, whose value $v=1 / \sqrt{3}$ is interpreted as a consequence of the average over the Fermi velocities. These quantities have been obtained previously by different methods [6]. By the same formalism we will determine the dispersion law for the gluons in the $\mu \rightarrow \infty$ limit. Finally we will couple the theory to external fermions. Integrating out the two-dimensional degrees of freedom we will get an effective theory describing NGB's and the in-medium fermions.

\section{Couplings to the Goldstone fields}

Let us recall the effective description of the CFL phase given in ref. [4]. The symmetry breaking is induced by the condensates

$$
\left\langle\psi_{a i}^{L} \psi_{b j}^{L}\right\rangle=-\left\langle\psi_{a i}^{R} \psi_{b j}^{R}\right\rangle=\gamma_{1} \delta_{a i} \delta_{b j}+\gamma_{2} \delta_{a j} \delta_{b i}
$$

where $\psi_{a i}^{L(R)}$ are Weyl spinors and a sum over spinor indices is understood. The indices $a, b$ and $i, j$ refer to $S U(3)_{c}$ and $S U(3)_{L}$ (or $\left.S U(3)_{R}\right)$ respectively. One then introduces $S U(3)$ matrix-valued fields $X$ and $Y$ transforming under the symmetry group $S U(3)_{c} \otimes S U(3)_{L} \otimes S U(3)_{R}$ as left-handed and righthanded fermions respectively. That is

$$
X \rightarrow g_{c} X g_{L}^{T}, \quad Y \rightarrow g_{c} Y g_{R}^{T} .
$$

Using the gauge freedom associated to $S U(3)_{c}$ it is possible to choose a gauge such that $X=Y^{\dagger}$. The condensates of eq. (16) break also $U(1)_{V} \otimes U(1)_{A}$ and as a consequence one also needs to introduce the related Goldstone fields. However we will not discuss these fields in this paper since all the calculations presented here are trivially extended to them [6].

The invariant coupling between fermions and Goldstone fields reproducing the symmetry breaking pattern of eq. (16) is proportional to

$$
\gamma_{1} \operatorname{Tr}\left[\psi_{L}^{T} X^{\dagger}\right] C \operatorname{Tr}\left[\psi_{L} X^{\dagger}\right]+\gamma_{2} \operatorname{Tr}\left[\psi_{L}^{T} C X^{\dagger} \psi_{L} X^{\dagger}\right]+\text { h.c. },
$$


and analogous relations for the right-handed fields. Here the spinors are meant to be Dirac spinors and $C=i \gamma^{2} \gamma^{0}$ is the charge-conjugation matrix. The trace is operating over the group indices of the spinors and of the Goldstone fields. Since the vacuum expectation value of the Goldstone fields is $\langle X\rangle=\langle Y\rangle=1$, we see that this coupling induces the correct breaking of the symmetry. In the following we will consider only the case $\gamma_{2}=-\gamma_{1} \propto \Delta / 2$, where $\Delta$ is the gap parameter. This choice corresponds to a condensate behaviour as the $(\mathbf{3}, \mathbf{3})$ representation of $S U(3)_{c} \otimes S U(3)_{L, R}$. This situations seems the one which is favorite by the dynamical analysis made in ref. [2]. Again, the calculations presented here can be easily extended to the more general situation where the condensate behaves as $(\mathbf{3}, \mathbf{3}) \oplus(\mathbf{6}, \mathbf{6})$. By our choice here the condensate has the form

$$
\left\langle\psi_{a i}^{L} \psi_{b j}^{L}\right\rangle=-\left\langle\psi_{a i}^{R} \psi_{b j}^{R}\right\rangle=\gamma_{1} \epsilon_{a b I} \epsilon_{i j I},
$$

and the invariant coupling of eq. (18) can be written as

$$
-\frac{\Delta}{2} \sum_{I=1,3} \operatorname{Tr}\left[\left(\psi X^{\dagger}\right)^{T} C \epsilon_{I}\left(\psi X^{\dagger}\right) \epsilon_{I}\right]
$$

where, now, also $\psi$ is considered as a $3 \times 3$ matrix and the matrix $\epsilon_{I}$ is defined as

$$
\left(\epsilon_{I}\right)_{a b}=\epsilon_{I a b} .
$$

Notice that for any $S U(3)$ matrix $g$ one has the identity

$$
g^{T} \epsilon_{I} g=\epsilon_{I^{\prime}}\left(g^{\dagger}\right)_{I^{\prime} I}
$$

Then it is easy to show that our expression for the coupling is equivalent to the one used by the authors of ref. [5]

$$
-\frac{\Delta}{2} \sum_{I=1,3} \operatorname{Tr}\left[\left(\psi X^{\dagger}\right)^{T} C \epsilon_{I}\left(\psi X^{\dagger}\right) \epsilon_{I}\right]=-\frac{\Delta}{2} \sum_{I, I^{\prime}=1,3} \operatorname{Tr}\left[\psi^{T} C \epsilon_{I} X_{I I^{\prime}} \psi \epsilon_{I^{\prime}}\right] .
$$

Since the transformation properties under the symmetry group of the fields at fixed Fermi velocity, as introduced in the previous section, do not differ from those of the quark fields, for both left-handed and right-handed fields we get the effective lagrangian density

$$
\mathcal{L}_{1}=\sum_{\vec{v}_{F}} \frac{1}{2}\left[\sum_{A=1}^{9}\left(\psi_{+}^{A \dagger} i V \cdot D \psi_{+}^{A}+\psi_{-}^{A \dagger} i \tilde{V} \cdot D \psi_{-}^{A}-\Delta_{A}\left(\psi_{-}^{A^{T}} C \psi_{+}^{A}+\text { h.c. }\right)\right)\right.
$$




$$
\left.-\Delta \sum_{I=1,3}\left(\operatorname{Tr}\left[\left(\psi_{-} X_{1}^{\dagger}\right)^{T} C \epsilon_{I}\left(\psi_{+} X_{1}^{\dagger}\right) \epsilon_{I}\right]+\text { h.c. }\right)\right]
$$

where we have introduced the fields

$$
\psi_{ \pm}=\frac{1}{\sqrt{2}} \sum_{A=1}^{9} \lambda_{A} \psi_{ \pm}^{A}
$$

and $\lambda_{a}(a=1, \ldots, 8)$ are the Gell-Mann matrices normalized as follows: $\operatorname{Tr}\left(\lambda_{a} \lambda_{b}\right)=2 \delta_{a b}$ and $\lambda_{9}=\sqrt{2 / 3}$ 1. Furthermore $\Delta_{1}=\cdots=\Delta_{8}=\Delta$ and $\Delta_{9}=-2 \Delta$ and $X_{1}=X-1$. Notice that the NGB fields couple to fermionic fields with opposite Fermi velocities. In this expression, as in the following ones, the field $\psi_{-}$is defined as $\psi_{+}$with $\vec{v}_{F} \rightarrow-\vec{v}_{F}$, and therefore it is not the same as the one defined in (8). In the previous lagrangian we have also separated the free quadratic terms in the fermionic fields. These terms can be rewritten in a more compact way by introducing the following fields

$$
\chi=\left(\begin{array}{c}
\psi_{+} \\
C \psi_{-}^{*}
\end{array}\right)
$$

It is important to realize that the fields $\chi$ and $\chi^{\dagger}$ are not independent variables. In fact, since we integrate over all the Fermi surface, the fields $\psi_{-}^{*}$ and $\psi_{+}$, appearing in $\chi$, appear also in $\chi^{\dagger}$ when $\vec{v}_{F} \rightarrow-\vec{v}_{F}$. In order to avoid this problem we can integrate over half of the Fermi surface, or, taking into account the invariance under $\vec{v}_{F} \rightarrow-\vec{v}_{F}$, we can simply integrate over all the sphere with a weight $1 / 8 \pi$ instead of $1 / 4 \pi$ :

$$
\sum_{\vec{v}_{F}}=\int \frac{d \vec{v}_{F}}{8 \pi}
$$

Then the first three terms in the lagrangian density (24) become

$$
\mathcal{L}_{0}=\int \frac{d \vec{v}_{F}}{8 \pi} \frac{1}{2} \sum_{A=1}^{9} \chi^{A \dagger}\left[\begin{array}{cc}
i V \cdot D & \Delta^{A} \\
\Delta^{A} & i \tilde{V} \cdot D^{*}
\end{array}\right] \chi^{A}
$$

so that, in momentum space the free fermion propagator is

$$
S_{A B}(p)=\frac{2 \delta_{A B}}{V \cdot p \tilde{V} \cdot p-\Delta_{A}^{2}}\left[\begin{array}{cc}
\tilde{V} \cdot p & -\Delta_{A} \\
-\Delta_{A} & V \cdot p
\end{array}\right] .
$$


We note explicitly that at this stage, since we have integrated out the degrees of freedom of the gluons in order to obtain the condensate appearing in eq. (24), the gluon field appearing in the covariant derivative in (28) has to be interpreted as an external field. From eq. (28) we can read also the fermionic current which is given by

$$
\begin{aligned}
J_{\mu}^{a} & =\frac{1}{4}\left(\sqrt{\frac{2}{3}} \sum_{a=1}^{8}\left(\chi^{9 \dagger}\left[\begin{array}{cc}
V_{\mu} & 0 \\
0 & -\tilde{V}_{\mu}
\end{array}\right] \chi^{a}+\text { h.c. }\right)\right. \\
& \left.+\sum_{b, c=1}^{8} \chi^{b \dagger}\left[\begin{array}{cc}
V_{\mu} g_{b a c} & 0 \\
0 & -\tilde{V}_{\mu} g_{b a c}^{*}
\end{array}\right] \chi^{c}\right)
\end{aligned}
$$

where

$$
g_{a b c}=d_{a b c}+i f_{a b c},
$$

and the symbols $d_{a b c}, f_{a b c}$ are defined, as usual, by $\lambda_{a} \lambda_{b}+\lambda_{b} \lambda_{a}=2 d_{a b c} \lambda_{c}+$ $\frac{4}{3} \delta_{a b}$ and $\lambda_{a} \lambda_{b}-\lambda_{b} \lambda_{a}=2 i f_{a b c} \lambda_{c}$.

Moreover, expanding the NGB fields

$$
X=\exp i\left(\frac{\lambda_{a} \Pi^{a}}{2 F}\right), \quad a=1, \cdots, 8,
$$

we get, considering terms up to the second order in the Goldstone fields:

$$
X_{1}=i \frac{\lambda_{a} \Pi^{a}}{2 F}-\frac{\lambda_{a} \lambda_{b} \Pi^{a} \Pi^{b}}{8 F^{2}} .
$$

Using these expressions, the trilinear coupling of the NGB's to the fermions arising from the last term in (24) can be written as

$$
\begin{aligned}
& \mathcal{L}_{\chi \chi \Pi}=\int \frac{d \vec{v}_{F}}{8 \pi} \frac{i \Delta}{2 F} \times \\
& \left(\sum_{a=1}^{8}\left(\chi^{9 \dagger}\left[\begin{array}{cc}
0 & -1 \\
1 & 0
\end{array}\right] \chi^{a} \frac{\Pi^{a}}{\sqrt{6}}+\text { h.c. }\right)-\sum_{a, b, c=1}^{8} d_{a b c} \chi^{a \dagger}\left[\begin{array}{cc}
0 & -1 \\
1 & 0
\end{array}\right] \chi^{b} \Pi^{c}\right)
\end{aligned}
$$

The quadrilinear coupling of two Goldstone bosons to two fermions is

$$
\mathcal{L}_{\chi \chi \Pi \Pi}=\int \frac{d \vec{v}_{F}}{8 \pi}\left\{\frac{4}{3} \sum_{a=1}^{8} \frac{\Delta}{16 F^{2}} \chi^{9 \dagger}\left[\begin{array}{cc}
0 & +1 \\
1 & 0
\end{array}\right] \chi^{9} \Pi^{a} \Pi^{a}\right.
$$




$$
\begin{aligned}
& +3 \sqrt{\frac{2}{3}} \sum_{a, b, c=1}^{8}\left(\frac{\Delta}{16 F^{2}} d_{a b c} \chi^{c \dagger}\left[\begin{array}{cc}
0 & +1 \\
1 & 0
\end{array}\right] \chi^{9} \Pi^{a} \Pi^{b}+\text { h.c. }\right) \\
& \left.-\sum_{a, b, c, d=1}^{8} \frac{\Delta}{16 F^{2}} h_{a b c d} \chi^{c \dagger}\left[\begin{array}{cc}
0 & -1 \\
1 & 0
\end{array}\right] \chi^{d} \Pi^{a} \Pi^{b}\right\}
\end{aligned}
$$

where

$$
h_{a b c d}=2 \sum_{p=1}^{8}\left(g_{c a p} g_{d b p}+d_{c d p} d_{a b p}\right)-\frac{8}{3} \delta_{a c} \delta_{d b}+\frac{4}{3} \delta_{c d} \delta_{a b} .
$$

\section{Gradient expansion}

We will treat the Goldstone bosons as external fields and we will perform a derivative expansion of the generating functional. This will give rise to the effective action for the NGB's. At the lowest order the relevant diagrams with two external NGB lines are the ones in Fig. 1. As previously discussed,

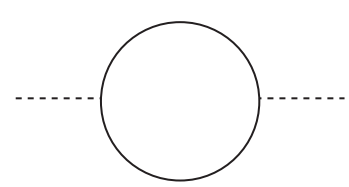

a)

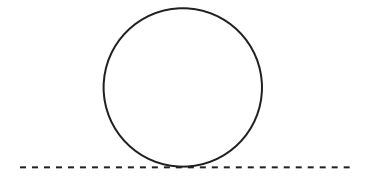

b)

Fig. 1 - One-loop diagrams in the gradient expansion. Dotted lines are the external fields, full lines are fermion propagators.

since the fermions live effectively near the Fermi surface, the four-dimensional momentum integration can be reduced to a two-dimensional one according to the formula

$$
\int \frac{d^{4} \ell}{(2 \pi)^{4}}=\frac{\mu^{2}}{\pi} \int \frac{d^{2} \ell}{(2 \pi)^{2}}
$$

where $d^{2} \ell=d \ell_{0} d \ell_{\|}$; notice that effectively the integration limits are $(-\mu,+\mu)$. In the Feynman rules one has also to take into account a factor 2 coming from the contribution of left-handed and right-handed fermions.

The tadpole diagram contributes only to the mass term and it is essential to cancel the external momentum independent term arising from the 
other diagram. Therefore, as expected, the mass of the NGB's is zero. The contribution at the second order in the momentum expansion is given by

$$
i \frac{21-8 \ln 2}{72 \pi^{2} F^{2}} \int \frac{d \vec{v}_{F}}{4 \pi} \sum_{a=1}^{8} \Pi^{a} V \cdot p \tilde{V} \cdot p \Pi^{a} .
$$

Integrating over the velocities and going back to the coordinate space we get

$$
\mathcal{L}_{\mathrm{eff}}^{\mathrm{kin}}=\frac{21-8 \ln 2}{72 \pi^{2} F^{2}} \sum_{a=1}^{8}\left(\dot{\Pi}^{a} \dot{\Pi}^{a}-\frac{1}{3}\left|\vec{\nabla} \Pi_{a}\right|^{2}\right) .
$$

We can now determine the decay coupling constant $F$ through the requirement of getting the canonical normalization for the kinetic term; this implies, non nova sed nove,

$$
F^{2}=\frac{\mu^{2}(21-8 \ln 2)}{36 \pi^{2}}
$$

a result already obtained by other authors with a different method.

In this formalism the origin of the goldstone velocity $1 / \sqrt{3}$ is a direct consequence of the integration over the Fermi velocity. Therefore it is completely general and applies to all the NGB's in the theory, including the ones associated to the breaking of $U(1)_{V}$ and $U(1)_{A}$; needless to say, higher order terms in the expansion $1 / \mu$ could change this result.

The breaking of the Lorentz invariance, exhibited by the pion velocity being different from one, can be seen also in the matrix element $\left\langle 0\left|J_{\mu}^{a}\right| \Pi^{b}\right\rangle$. This can be evaluated using the Feynman diagram of Fig. 2. The result,

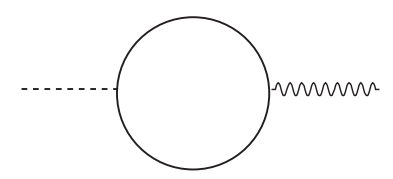

Fig. 2 - Diagram contributing to the matrix element of the fermionic current between the CFL vacuum and the single NGB state. The dotted line represents the Goldstone boson, the wavy line the current and the internal lines correspond to fermions.

after the integration over the Fermi velocity is

$$
\left\langle 0\left|J_{\mu}^{a}\right| \Pi^{b}\right\rangle=i F \delta_{a b} \tilde{p}_{\mu}, \quad \tilde{p}^{\mu}=\left(p^{0}, \frac{1}{3} \vec{p}\right)
$$


where we have made use of the previous result for $F$. It is interesting to notice that the current is conserved, as the dispersion law for the NGB's has the form $p \cdot \tilde{p}=p_{0}^{2}-1 / 3|\vec{p}|^{2}=0$.

\section{Dispersion law for the gluons}

The dispersion law for the in-medium gluons can be derived by the same formalism used in the previous section. It is equivalent to consider the polarization tensor $\Pi^{\mu \nu}(p)$. Its one-loop contribution is obtained by computing diagrams analogous to the ones in Fig. 1 (with external lines representing currents $J_{\mu}^{a}, J_{\nu}^{b}$ as given by (30)). The result of the first diagram (Fig.1 a) is:

$$
\left.\Pi_{a b}^{\mu \nu}(p)\right|_{1 a}=\Pi_{a b}^{\mu \nu}(0)+\delta \Pi_{a b}^{\mu \nu}(p)
$$

where

$$
\begin{aligned}
& \Pi_{a b}^{\mu \nu}(0)=\frac{\mu^{2} g_{s}^{2}}{4 \pi} \delta_{a b} \int \frac{d \vec{v}_{F}}{4 \pi} \Sigma^{0, \mu \nu}, \\
& \delta \Pi_{a b}^{\mu \nu}(p)=\frac{\mu^{2} g_{s}^{2}}{4 \pi} \delta_{a b} \int \frac{d \vec{v}_{F}}{4 \pi} \Sigma^{\mu \nu}
\end{aligned}
$$

and

$$
\begin{gathered}
\Sigma^{0, \mu \nu}=k_{1}\left(V^{\mu} V^{\nu}+\tilde{V}^{\mu} \tilde{V}^{\nu}\right)+k_{2}\left(V^{\mu} \tilde{V}^{\nu}+\tilde{V}^{\mu} V^{\nu}\right) \\
\Sigma^{\mu \nu}=a\left(V^{\mu} V^{\nu} \frac{(\tilde{V} \cdot p)^{2}}{\Delta^{2}}+\tilde{V}^{\mu} \tilde{V}^{\nu} \frac{(V \cdot p)^{2}}{\Delta^{2}}\right)+b\left(V^{\mu} \tilde{V}^{\nu}+\tilde{V}^{\mu} V^{\nu}\right) \frac{V \cdot p \tilde{V} \cdot p}{\Delta^{2}} .
\end{gathered}
$$

In the previous formulas we have included terms up to the second order in momenta. The coefficients are given by

$$
\begin{aligned}
a & =\frac{1}{108 \pi}\left(31-\frac{32}{3} \ln 2\right) \\
b & =\frac{10}{108 \pi}\left(1-\frac{8}{3} \ln 2\right) \\
k_{1} & =\frac{3}{2 \pi} \\
k_{2} & =-\frac{1}{9 \pi}(3+4 \ln 2) .
\end{aligned}
$$

To this result one should add the contribution arising from the tadpole diagram of Fig. 1b (with, also in this case, external lines representing fermionic 
currents $\left.J_{\mu}^{a}, J_{\nu}^{b}\right)$. This diagram arises from the last term in (14). It can be computed by performing first the integration over the energy $\ell_{0}$ and subsequently over the longitudinal momentum $\ell_{\|}$[6]. This integration introduces an extra factor $\mu$ besides the $\mu^{2}$ factor arising from the integration over the Fermi surface. The result is independent on the external momentum $p$ and is given by:

$$
\left.\Pi_{a b}^{\mu \nu}(0)\right|_{1 b}=\Delta \Pi_{a b}^{\mu \nu}(0)=\frac{\mu^{2} g_{s}^{2}}{4 \pi} \delta_{a b} \int \frac{d \vec{v}_{F}}{4 \pi} \Sigma_{B}^{0, \mu \nu}
$$

with

$$
\Sigma_{B}^{0, \mu \nu}=k_{1}\left[2 g^{\mu \nu}-V^{\mu} \tilde{V}^{\nu}-V^{\nu} \tilde{V}^{\mu}\right] .
$$

From $\Pi_{a b}^{\mu \nu}(0)+\Delta \Pi_{a b}^{\mu \nu}(0)$ we can read the one-loop contributions to the Debye and Meissner mass

$$
m_{D}^{2}=g_{s}^{2} F^{2}=\frac{\mu^{2} g_{s}^{2}}{36 \pi^{2}}(21-8 \ln 2)
$$

and

$$
m_{M}^{2}=\frac{\mu^{2} g_{s}^{2}}{\pi^{2}}\left(-\frac{11}{36}-\frac{2}{27} \ln 2+\frac{1}{2}\right)=\frac{m_{D}^{2}}{3},
$$

where the first two terms are the result of the diagram of Fig.1a and the last one is the result of the diagram of Fig. 1b (this contribution is called the bare Meissner mass in [6]). These results agree with the findings of other authors [6], [10]. In particular $m_{M}^{2}=v^{2} m_{D}^{2}$, where $v$, the NGB velocity, is equal to $1 / \sqrt{3}$.

It may be noted, in passing, that the polarization tensor $\left.\Pi_{a b}^{\mu \nu}(p)\right|_{1 a+1 b}$ satisfies a Ward identity in the soft NGB limit. In fact, let us consider the amplitude $p_{\nu} \Pi_{a b}^{\mu \nu}(p)$, with $\Pi_{a b}^{\mu \nu}(p)=\left.\Pi_{a b}^{\mu \nu}(p)\right|_{1 a+1 b}$, in the $p \rightarrow 0$ limit. In this kinematical regime it should be dominated by the massless NGB pole and it should be proportional to $\left\langle 0\left|J_{\mu}^{a}\right| \Pi^{b}\right\rangle=i F \delta_{a b} \tilde{p}_{\mu}$. In the $p \rightarrow 0$ limit we find indeed

$$
p_{\nu} \Pi_{a b}^{\mu \nu}(p) \propto \tilde{p}^{\mu} .
$$

For this behaviour to be found it is essential to include both diagrams of Fig.1.

In order to derive the dispersion law for the gluons, we first write the equations of motion for the gluon field $A_{\mu}^{b}$ in momentum space and highdensity limit:

$$
\Pi_{a b}^{\nu \mu} A_{\mu}^{b}=0
$$


from which we obtain

$$
p_{\nu} \Pi_{a b}^{\nu \mu} A_{\mu}^{b}=0,
$$

i.e. for all the gluons:

$$
p^{0} A_{0}=\frac{1}{3}(\vec{p} \cdot \vec{A})+\ldots,
$$

where the ellipsis denotes terms of the third order in the gluon momenta that, consistently with our approximation, we neglect. Substituting this result in (56) taken for $\nu=0$ one gets the dispersion law for the time-like gluons $A_{0}^{b}$

$$
3 \alpha_{1} E^{2}-\alpha_{1}|\vec{p}|^{2}=m_{D}^{2}
$$

with the factor 3 arising from the coupling of $A_{0}$ to $A_{i}$ in the equation of motion and the use of eq. (58). This gives

$$
p^{0}= \pm E_{A_{0}}, \quad E_{A_{0}}=\frac{1}{\sqrt{3}} \sqrt{|\vec{p}|^{2}+\frac{m_{D}^{2}}{\alpha_{1}}},
$$

with

$$
\alpha_{1}=\frac{\mu^{2} g_{s}^{2}}{6 \Delta^{2} \pi}(a-b)=\frac{\mu^{2} g_{s}^{2}}{216 \Delta^{2} \pi^{2}}\left(7+\frac{16}{3} \ln 2\right) .
$$

Notice that the rest mass of $A_{0}^{b}$ is given by

$$
m_{A_{0}}^{R}=\frac{m_{D}}{\sqrt{3 \alpha_{1}}}=\sqrt{6 \frac{21-8 \ln 2}{21+16 \ln 2}} \Delta \approx 1.70 \Delta .
$$

This result may appear surprising since the Debye and the Meissner masses are of the order $g_{s} \mu$. However one should take into account that the renormalization of the coefficient of $E^{2}$ (that is $\alpha_{1}$ ), arising from the polarization tensor, redefines the physical mass by a factor proportional to $\Delta / g_{s} \mu$ and the coupling constant $g_{s}$ as well. Actually there are various different "masses" which can be defined, apart from the rest mass, when we consider the low momentum limit. For instance we can consider the inverse of the penetration length, $m_{A_{0}}^{P}$, defined for $E \rightarrow 0$ as the ratio between the mass term and the coefficient of $|\vec{p}|^{2}$. This mass is given by

$$
m_{A_{0}}^{P}=\sqrt{3} m_{A_{0}}^{R} \approx 2.94 \Delta .
$$


We can finally define a third mass, sometimes called effective mass, $m^{*}$, by considering the ratio between the spatial momentum and the velocity, i.e. by looking at the following expression

$$
\vec{v}=\frac{\partial E}{\partial \vec{p}}=\frac{\vec{p}}{m^{*}(p)}
$$

in the limit $\vec{p} \rightarrow 0$, that is

$$
m^{*}=m^{*}(0) .
$$

The meaning of this last mass for ordinary particles would be the coefficient appearing in the kinetic energy term. One obtains

$$
m_{A_{0}}^{*}=\sqrt{\frac{3}{\alpha_{1}}} m_{D}=3 m_{A_{0}}^{R} \approx 5.10 \Delta .
$$

Let us now turn to the dispersion law for $\overrightarrow{A^{a}}$; we consider (56) for $\nu=i$ and use again (58). We introduce longitudinal and transverse components:

$$
\begin{aligned}
A_{L}^{i a} & =\frac{\vec{p} \cdot \vec{A}^{a}}{|\vec{p}|^{2}} p^{i} \\
A_{T}^{i a} & =A^{i a}-A_{L}^{i a},
\end{aligned}
$$

and we project the equations of motion along the longitudinal and transverse direction. The results for the longitudinal and transverse cases are respectively

$$
\begin{aligned}
\alpha_{1} E^{2}-\alpha_{2} \frac{|\vec{p}|^{2}}{3} & =\frac{m_{D}^{2}}{3} \\
\alpha_{1} E^{2}-\alpha_{3}|\vec{p}|^{2} & =\frac{m_{D}^{2}}{3}
\end{aligned}
$$

from which

$$
\begin{aligned}
& E_{A_{L}}=\frac{1}{\sqrt{3}} \sqrt{|\vec{p}|^{2} \frac{\alpha_{2}}{\alpha_{1}}+\frac{m_{D}^{2}}{\alpha_{1}}} \\
& E_{A_{T}}=\sqrt{|\vec{p}|^{2} \frac{\alpha_{3}}{\alpha_{1}}+\frac{m_{D}^{2}}{3 \alpha_{1}}}
\end{aligned}
$$


with

$$
\begin{aligned}
\alpha_{2} & =\frac{\mu^{2} g_{s}^{2}}{30 \Delta^{2} \pi^{2}}(a-9 b)=-\frac{\mu^{2} g_{s}^{2}}{3240 \Delta^{2} \pi^{2}}\left(59-\frac{688}{3} \ln 2\right) \\
\alpha_{3}=-\frac{\mu^{2} g_{s}^{2}}{30 \Delta^{2} \pi^{2}}(a+b) & =-\frac{\mu^{2} g_{s}^{2}}{3240 \Delta^{2} \pi^{2}}\left(41-\frac{112}{3} \ln 2\right) .
\end{aligned}
$$

We get easily the following results

$$
m_{A_{L}}^{R}=m_{A_{T}}^{R}=m_{A_{0}}^{R}=\frac{m_{D}}{\sqrt{3 \alpha_{1}}} \equiv m_{R} .
$$

Since $\alpha_{3}$ is large and negative, the inverse of the penetration lenght looses its meaning for $A_{T}$. On the other hand we get

$$
m_{A_{L}}^{P}=\frac{m_{D}}{\sqrt{\alpha_{2}}}=\sqrt{\frac{3 \alpha_{1}}{\alpha_{2}}} m^{R} \approx 3.73 \Delta .
$$

We get also

$$
\begin{aligned}
& m_{A_{L}}^{*}=\frac{\sqrt{3 \alpha_{1}}}{\alpha_{2}} m_{D}=3 \frac{\alpha_{1}}{\alpha_{2}} m^{R} \approx 8.19 \Delta \\
& m_{A_{T}}^{*}=\frac{1}{\sqrt{3}} \frac{\sqrt{\alpha_{1}}}{\alpha_{3}} m_{D}=\frac{\alpha_{1}}{\alpha_{3}} m^{R} \approx-18.04 \Delta .
\end{aligned}
$$

Since $m_{A_{T}}^{*}$ is negative, the spectrum of the quasi-particles associated to the transverse gluons has a maximum for $|\vec{p}|=0$, which means that at very small temperatures, which is the limit in which we work, these quasi-particles are unlikely to be produced: this situation reminds the spectrum of the elementary excitations of superfluid $H e^{4}$ that, besides the phonon and roton parts, presents a maximum for intermediate momenta.

It can be useful to note that all these mass scales are determined in terms of the common rest mass $m^{R}$ and of the ratios $\alpha_{1} / \alpha_{2} \approx 1.61$ and $\alpha_{1} / \alpha_{3} \approx-10.61$

\section{In-medium fermions}

To obtain the effective description for the in-medium fermions, let us introduce for both the left-handed and right-handed fermion fields $\chi\left(=\chi_{L}, \chi_{R}\right)$ external fields $\Psi\left(=\Psi_{L}, \Psi_{R}\right)$. By defining

$$
\eta_{ \pm}=P_{ \pm} \Psi
$$


and

$$
\Phi=\left(\begin{array}{c}
\eta_{+} \\
C \eta_{-}^{*}
\end{array}\right),
$$

with $P_{ \pm}$given in (10), we can couple the external field $\Phi$ to the field $\chi$ (for simplicity we consider here a single Fermi field). Then we have a lagrangian density

$$
\mathcal{L}=\mathcal{L}_{0}+\mathcal{L}_{\text {source }}=\int \frac{d \vec{v}_{F}}{8 \pi}\left(\chi^{\dagger} S^{-1} \chi+\chi^{\dagger} \Phi+\Phi^{\dagger} \chi\right)
$$

with

$$
S^{-1}=\frac{1}{2}\left[\begin{array}{cc}
i V \cdot D & \Delta \\
\Delta & i \tilde{V} \cdot D^{*}
\end{array}\right] .
$$

Integrating over the field $\chi$, or eliminating it through the equations of motion, we get an effective action for $\Phi$

$$
\mathcal{L}_{\text {eff }}=\int \frac{d \vec{v}_{F}}{8 \pi} \frac{2}{\Delta^{2}} \Phi^{\dagger}\left[\begin{array}{cc}
i \tilde{V} \cdot D^{*} & -\Delta \\
-\Delta & i V \cdot D
\end{array}\right] \Phi,
$$

where we have made an expansion for low momenta and small coupling constant. By re-expressing $\Phi$ in terms of $\Psi$ and performing the integration over the Fermi velocity we get

$$
\mathcal{L}_{\text {eff }}=\left(\bar{\Psi}, \bar{\Psi}_{c}\right)\left[\begin{array}{cc}
i \hat{\gamma} \cdot D^{*} & \Delta \\
\Delta & i \hat{\gamma} \cdot D
\end{array}\right]\left(\begin{array}{c}
\Psi \\
\Psi_{c}
\end{array}\right),
$$

where we have defined

$$
\Psi_{c}=C \bar{\Psi}^{T}
$$

and

$$
\hat{\gamma}^{\mu}=\left(\gamma^{0},-\frac{1}{3} \gamma^{i}\right),
$$

and we have scaled the field as $\Psi$

$$
\Psi \rightarrow \frac{1}{\sqrt{2} \Delta} \Psi
$$

One can notice that the origin of the $1 / 3$ in the lagrangian is again due to the average on the Fermi velocity.

The dispersion relation for the field $\Psi$ is

$$
\tilde{p}^{2}-\Delta^{2}=0, \quad \tilde{p}^{\mu}=\left(p^{0}, \frac{1}{3} \vec{p}\right),
$$


giving rise to

$$
p^{0}= \pm E, \quad E=\frac{1}{3} \sqrt{|\vec{p}|^{2}+9 \Delta^{2}} .
$$

We see that the rest mass is given by $\Delta$. The velocity of in-medium fermions is

$$
\vec{v}=\frac{\partial E}{\partial \vec{p}}=\frac{1}{3} \frac{\vec{p}}{\sqrt{|\vec{p}|^{2}+9 \Delta^{2}}} .
$$

and therefore, using the same definition as in the previous Section for $m^{*}$

$$
m^{*}=9 \Delta
$$

It is tempting, even if not reliable within our approximation, to consider the large $|\vec{p}|$ limit; for $|\vec{p}| \gg 3 \Delta$ one would get

$$
|\vec{v}|=\frac{1}{3}
$$

to be compared to the velocity of the NGB's given by $1 / \sqrt{3}$.

\section{Conclusions}

After a presentation of the basic points of the formalism, we have discussed the couplings of the goldstones and their effective lagrangian. We have written down explicitly the needed trilinear couplings of the goldstones to the fermions and the quadrilinear couplings of two goldstones to two fermions. To explicitly obtain the parameters of the effective action for the goldstones we have performed a derivative expansion of the generating functional. In this way we obtain the known results at one loop order. The value of the velocity of the goldstones appears explicitly as following from the integration over the Fermi velocity. We have then discussed the in-medium gluon polarization tensor. At the one loop order the formalism reproduces the correct values for the Debye and Meissner masses. The dispersion laws for both scalar and vector gluons follow from the gluon field equations of motion in the limit of high density; from them the various masses of the scalar, longitudinal and transverse gluons have been computed, i.e. the rest mass, the effective masses and the inverse of the penetration lenght. A main development has been the derivation of the effective description for in-medium fermions. We 
have introduced fermion external fields, both left- and right-handed, to be coupled to the dynamical fermion fields. After integrating over the dynamical fermions we have obtained an effective action and the fermionic dispersion relation in terms of the gap parameter. This provides for a value of the in-medium fermion mass and shows again the role played by the integration over the Fermi velocity.

The theory of color-flavor locking, as developed and studied by many authors, has undoubtedly an intrinsic beauty and the high density fermionic formalism used here provides for a simple and appealing description. It remains to be seen to which physical situations of matter under extreme conditions the theory can usefully be applied, most probably by including additional perturbations.

AKNOWLEDGMENTS We would like to thank Krishna Rajagopal for an illuminating discussion about a previous version of this paper.

\section{References}

[1] B. Barrois, Nucl. Phys. B129, 390 (1977); S. Frautschi, Proceedings of workshop on hadronic matter at extreme density, Erice 1978; D. Bailin and A. Love, Phys. Rept. 107, 325 (1984); M. Alford, K. Rajagopal and F. Wilczek, Phys. Lett. B422, 247 (1998); R. Rapp, T. Schäfer, E. V. Shuryak and M. Velkovsky, Phys. Rev. Lett. 81, 53 (1998); T. Schäfer and F. Wilczek, Phys. Rev. Lett. 82, 3956 (1999).

[2] M. Alford, K. Rajagopal and F. Wilczek, Nucl. Phys. B537 443 (1999), hep-ph/9804403.

[3] R. Rapp, T. Schäfer, E. V. Shuryak and M. Velkovsky, Annals Phys. 280, 35 (2000); D. K. Hong, M. Rho and I. Zahed, Phys. Lett. B468, 261 (1999); M. Alford, J. Berges and K. Rajagopal, Phys. Rev. Lett. 84, 598 (2000); T. Schäfer, Nucl. Phys. B575, 269 (2000); C. Manuel and M. H. Tytgat, Phys. Lett. B479, 190 (2000), and hep-ph/0010274; D. K. Hong, T. Lee and D. Min, Phys. Lett. B477, 137 (2000); M. Rho, E. Shuryak, A. Wirzba and I. Zahed, Nucl. Phys. A676, 273 (2000); D. H. Rischke, Phys. Rev. D62, 054017 (2000); D. K. Hong, 
hep-ph/0006105; T. Schäfer, nucl-th/0007021. M. A. Nowak, M. Rho, A. Wirzba and I. Zahed, hep-ph/0007034.

[4] R. Casalbuoni and R. Gatto, Phys. Lett. B464 111 (1999), hep-ph/9908227.

[5] M. Rho, A. Wirzba and I. Zahed, Phys. Lett. B473 126 (2000), hep-ph/9910550.

[6] D.T. Son and M.A. Stephanov, Phys. Rev. D61 074012 (2000), hep-ph/9910491, erratum: Phys. Rev. D62 059902(E) (2000), hep-ph/0004095

[7] D. K. Hong, Phys. Lett. B473 (2000) 118, . hep-ph/9812510;

D. K. Hong, Nucl. Phys. B582 (2000) 451, hep-ph/9905523.

[8] S. R. Beane, P. F. Bedaque and M. J. Savage, Phys. Lett. B483 (2000) 131, hep-ph/0002209.

[9] N. Isgur and M.B. Wise, Phys. Lett. B232 (1989) 113 and Phys. Lett. B237 (1990) 527; E. Eichten and B. Hill, Phys. Lett. B234 (1990) 511; H. Georgi, Phys. Lett. B249 (1990) 447; for a recent review see A. V. Manohar and M.B. Wise Heavy Quark Physics, Cambridge University Press (2000).

[10] K. Zarembo, Phys. Rev. D62, 054003 (2000) hep-ph/0002123. 